\title{
A SHORT MESSAGE SERVICE BASED DESIGN FOR A PORTABLE TELEALARM DEVICE
}

\author{
Ren-Guey LeE ${ }^{1}$ and KuANG-ChIUNG CHANG ${ }^{2}$ \\ ${ }^{1}$ Department of Electronic Engineering, National Taipei University of Technology, Taipei \\ ${ }^{2}$ Department of Electrical Engineering, Lunghwa University of Science and Technology \\ Tauyan, Taiwan
}

ABSTRACT

This paper presents an SMS based design in the GSM system for a portable, light weighted, and small sized TeleAlarm device. The device is composed of a transmitter and a controller. When an emergent situation such as a stroke or a fall occurs, the user only needs to push a button to trigger the controller. The controller automatically sends text messages stored in its database through the transmitter to specified mobile phone numbers for help. The SMS uses only the control channels in the GSM system to transfer the message, which enables the receiving-end user to receive it even during a call. An experimental test shows that a complete message transmission only needs 2.949 seconds in average. The design is comvenient to elderly people who may live alone.

Biomed Eng Appl Basis Comm. 2002 (June): 14: 109-114.

Key Words: GSM, SMS. GPS

\section{INTRODUCTION}

One of the major problems of aging society is the healthcare of elderly people. Since a telecare system is now able to provide medical advice or treatment at home on the scene without the necessity for healthcare providers or patients to travel, it may produce benefits for public care services [1-5]. Therefore, the use of the telecare systems to support the elderly people living alone at home is suggested in a lot of studies, e.g., $[6,7]$ and references there in. Home could be a danger place. In [8] Williams et al. propose an integrated telecare system to reduce the risk of merely using telecare technology at home. The integrated telecare system uses sensors, monitors, and intelligence controllers to track the actions of the patient and control the potentially hazardous situations. The design meets a particular client's special needs, but is expensive.

Received: Nov. 13, 2001; Accepted: April 29, 2002

Correspondence: Dr. Kuang-Chiung Chang,

Department of Electrical Engineering, Lunghwa

University of Science and Technology, 300. Wan-Shou

Rd., Sec. 1, Kueishan, Tauyan, 33327 Taiwan
When an accident such as a heavy fall or an unexpected illness such as a stroke occurs, the elderly living-alone may not be able to make a complete call for emergency assistance. In this case, a device must be used to compensate for the incompletion of the telecare systems. The Global System for Mobile Communications (GSM) based telemedicine system has been studied [9-11] and is often seen as an economically attractive method of healthcare in the areas where standard telephone services are not universally available. Such an area is similar to home of the injured who can not reach or use a telephone at that moment. The study proposes a Short Message Service (SMS) based design in the GSM system for a portable, light weighted, and small sized TeleAlarm device to solve this problem.

The paper is organized as follows: a basic concept about the operation of the GSM system is given in section 2. In addition, the SMS transmission properties are also included. A hardware design and a software design of the SMS based TeleAlarm device are described in section 3 and section 4 respectively. The performance of the TeleAlarm device is tested and the result is shown in section 5. Finally, concluding remarks are made in section 6 . 


\section{OVERVIEW OF THE SMS IN THE GSM SYSTEM}

In this section, a brief description of the GSM system and the SMS transmission characteristics is given. For further details concerning this subject, good references are $[12,13]$.

A simplified architecture of the GSM system is shown in Fig. 1. The mobile station (MS) consists of a subscriber identity module (SIM) card and mobile equipment (ME) such as a mobile phone. In the base station subsystem, the base station controller (BSC) uses the frequency division multiple access (FDMA) technique randomly assigns an unassigned channel or central carrier frequency to one of its base transceiver station (BTS). The BTS divides the assigned channel into several time slots by using the time division multiple access (TDMA) technique. Each time slot is provided to an active MS. The mobile service switching center (MSC) is located between the BSC and the other networks such as a public switched telephone network (PSTN) or an integrated services digital network (ISDN). Through the MSC, it is possible to transfer a voice message from an MS to an MS or to a phone. It is also possible to transfer a text or data message between MSs, if the MSC is connected with a short message service center (SMSC). The short message is of the form

"mobile phone number" "text message"

in which the text message is usually of a maximum length of 140 characters in Taiwan area, i.e. short message. In general, the maximum length of the short message (1), including the 4 double quotation marks and 1 space needed, 10 digits of the mobile phone number, and text message, is 155 characters.

When the SMSC receives the short message, it sends only once the text message to the specified MS that the short message assigns. In the transferring, the SMSC provides a store and forward function for short

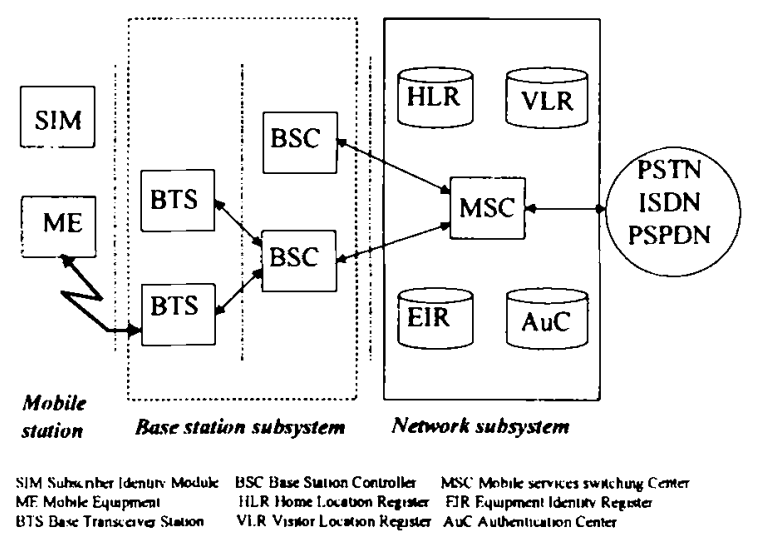

Fig. 1 A simplified architecture of the GSM system. message. It confirms each exchange of short message by giving a confirmation message. However, the confirmation does not guarantee the specified MS to get a clear text message. As stated above, the SMSC only sends the short message once, it may be lost when the specified MS is within a weak GSM coverage area. In this case, there is no way to recover it since the SMSC will clear the stored short message, when it gets the confirmed signal from the specified MS.

The SMS uses only control channels to send the short messages, which enables the receiving-end user to receive the messages even it has a call in progress. The transmission of short message can be divided into two types: the broadcasting services and the point to point services. The broadcasting service provides multicast message transmission to the groups of terminals and is usually used by companies for commercial purposes. On the contrary, the point to point service only transfers a message to a specified terminal. In general, there are only few numbers that one can call for help when an emergency occurs. It is clear that the broadcasting service does not meet our needs.

From the brief review of the SMS above, it is clear that there are two advantages of the SMS. First, the SMS uses control channels to transfer the short messages i.e., it does not need to establish a voice truck as in the public phone systems. This property can be viewed as the short message having the highest priority of message transmission except control commands in the GSM network. Second, the SMS provides for confirmed messages transferring. The confirmation enables receiving-end users to get a clear text message as long as both of the transmitting-end user and receiving-end user are within the 'good' GSM coverage area. The advantages make the SMS particularly useful once the emergency occurs.

\section{HARDWARE DESIGN}

The aim of the SMS based TeleAlarm device design is to provide a push button triggered emergent

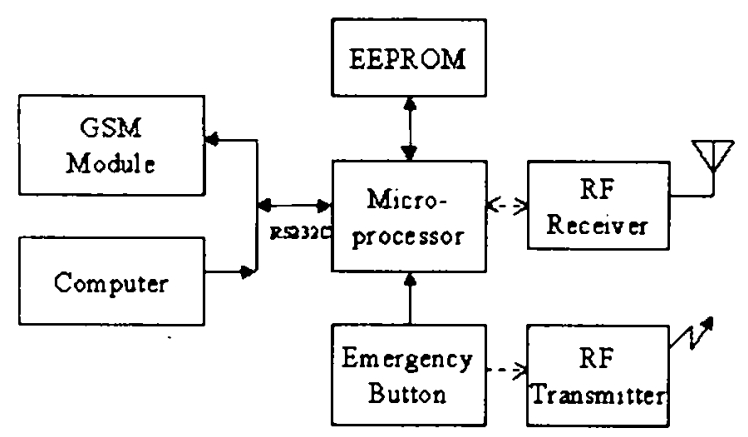

Fig. 2 The hardware block diagram. 
message sending device. The hardware block diagram is shown in Fig. 2, in which the GSM module and the microprocessor $89 \mathrm{C} 2051$ play the roles of the transmitter and the controller respectively.

As the GSM module equipped with a keyboard, a speaker, and a microphone, it can be a mobile phone as well. Under the consideration that a user may press wrong keys or may not be able to send a complete short message in some real emergent cases, the keyboard is replaced with only a push button. The microphone and speaker are omitted since the user does not need hear and speak in the SMS. Although the weight of the GSM module is very light, the push button can be replaced with a commercialized home radio frequency transmitter and receiver set that provides an ON/OFF signal for further reduction of the weight that a user should carry. The circuit board is shown in Fig. 3. The circuit board is packed into a box of $10 \mathrm{~cm}$ in length, $5 \mathrm{~cm}$ in width, and $2 \mathrm{~cm}$ in height and the total weight is about $600 \mathrm{~g}$.

As being the controller, the microprocessor controls the short messages sending procedure of the GSM module. The short messages are pre-stored as a database in the external electrical erased programmable read only memory (EEPROM) that connected with the microprocessor. The memory size of EEPROM is $4 \mathrm{~K}$ bytes that can be used to store about 25 sets of short messages (Each short message is of the maximum length of 155 characters). The memory size can be enlarged, if the 25 sets are not enough. Since the design avoids using the keyboard, a device, such as a personal computer (PC), must be used to establish or modify the short message through the microprocessor. Both of the GSM module and the microprocessor provide only one port of RS232C interface. Once they are connected with each other, as they should be, a diffi-

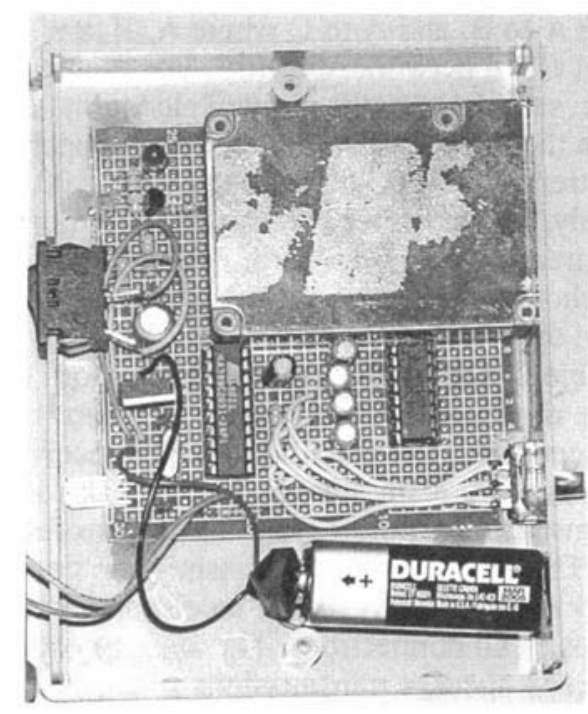

Fig. 3 The circuit board of the TeleAlarm device. culty raises with the lack of a second port of the RS232C interface on the microprocessor for the connection of the PC to establish or modify the short messages in the EEPROM. This problem will be solved by a software design in the next section.

\section{SOFTWARE DESIGN}

The hardware design of the TeleAlarm device described in the previous section leaves two major problems that the software in the microprocessor should overcome. One of the problems is how to distinguish the given commands between the GSM module for emergent help and the PC for the request of establishing or modifying the short messages in the EEPROM. This problem is due to use the same RS232C interface port on the microprocessor to connect with both of the GSM module and the computer. The other problem is how to send the short message when the emergency push button is pressed.

Before solving these problems, a brief review of the short message transmission format will assist in clarifying the idea of design. The SMS in the GSM system can be divided into three modes, namely, the text mode, the block mode, and the protocol data unit (PDU) mode. Only the text mode is used in the design and is discussed. To send a short message in the text mode, a format called Extend AT command should be used. The Extend AT command format in Visual Ba$\operatorname{sic}(B)$ is of the form

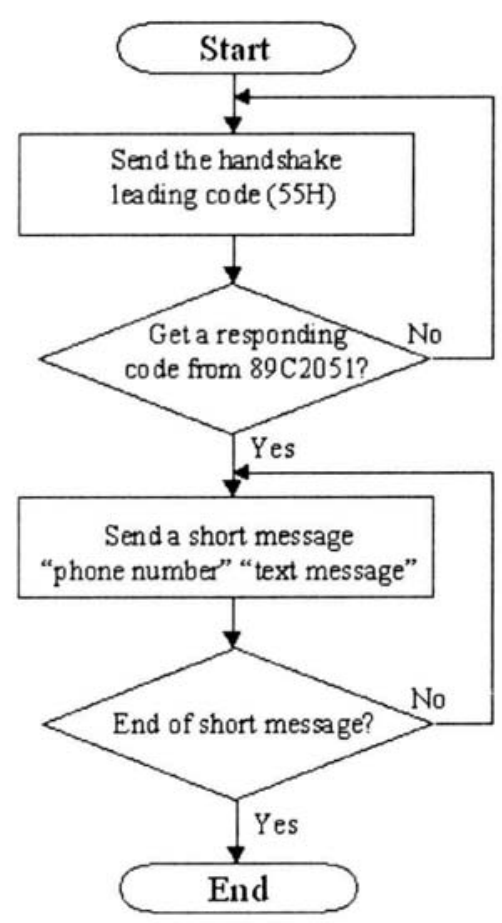

Fig. 4 The flowchart for storing the short messages. 


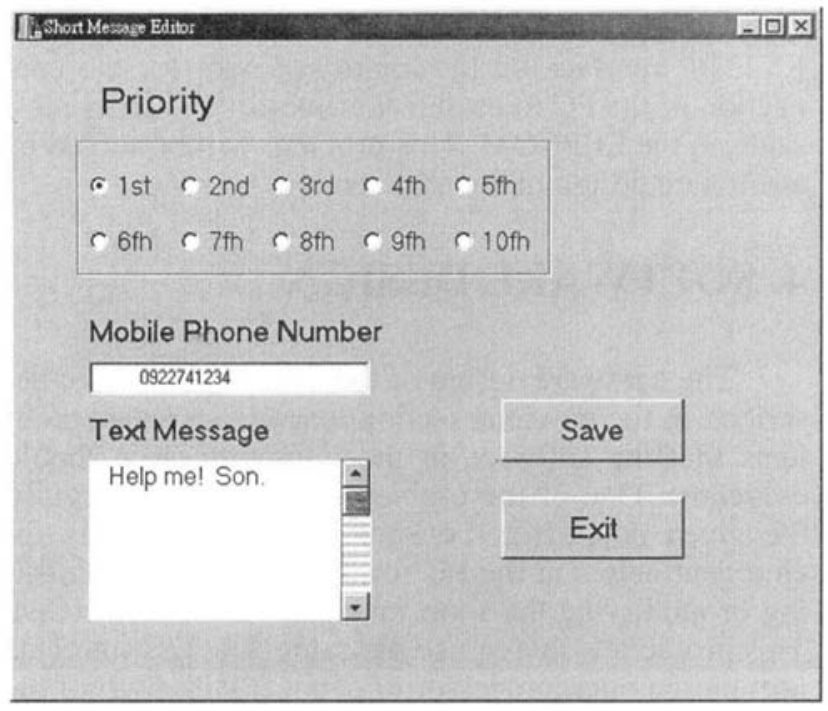

Fig. 5 The dialogue screen for storing the short messages.

"AT + command = mobile phone number" Chr(13) "text message" $\wedge \mathrm{Z}$

in which 'command' is one of the short message commands listed in Table 1. For instance, the command

"AT + CMGS = 0922741234" \& Chr(13) \& "Help me! Son." $\mathrm{Z}$

makes the SMSC to send the text message "Help me! Son.' to the MS numbered 0922741234 .

We are now ready to solve the first problem. The solution is given in Fig. 4. To establish or modify the short messages in the EEPROM, the program in the PC end starts with sending a handshake leading code. Since the structure of $55 \mathrm{H}$ in binary form, 01010101 , makes it one of the most noninterfered codes, we choose the $55 \mathrm{H}$ as the handshake leading code. The handshake leading code is the prelude that makes the microprocessor be able to check the connection of its RS232C interface port. The microprocessor gives a responding code when it is ready to receive and to record the new short messages. The PC sends the new short messages in the form of (1) after it gets the responding code. The PC does not send any Extend AT commands in this process. Hence, the GSM module is not activated, even though it also receives the short messages at the same time. The dialogue screen of the program in the PC is shown in Fig. 5. It allows the user to assign the priority of the short message, modify a recorded short message and store a new short message.

The flowchart of the program in the microprocessor is shown in Fig. 6. The program determines the connection of the microprocessor by checking whether it receives the handshake leading code or not. If not, the program goes to a standby mode until it senses that the emergency button is pressed. When the program receives a triggering signal from the emergency button, it adds the necessary commands into the recorded short

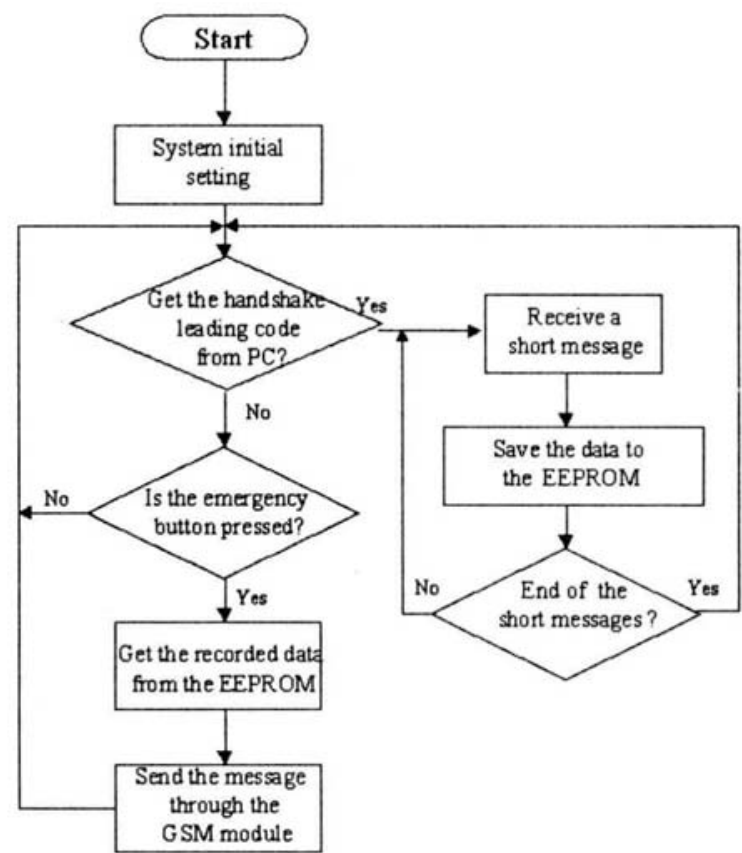

Fig. 6 The flowchart for sending the short messages.

message to develop the format in (2) and send it to the SMSC through the GSM module. One short message is transmitted at a time depending on the priority. The process can be made repetitively according to the user's need.

\section{AN EXPERIMENTAL RESULT}

The performance of the TeleAlarm device is tested under three different route connections, say, $\mathrm{A}$ to itself, $A$ to $B$, and $A$ to $C$ where $A, B$, and $C$ are the different GSM network operators. For each connection test, the signal strength of the TeleAlarm device remains in the same level while the MS is under received signal strength of $-50,-60,-70,-80,-90,-100 \mathrm{dBm}$, respectively. Under each particular condition of received signal strength, the TeleAlarm device sends 10 times of the short message to the same mobile phone with different operator's SIM card.

Table 2 shows the results. Two short messages are lost over 180 transmissions of short messages. Both occur under the condition that the received signal strength is $-100 \mathrm{dBm}$ and route connection is $\mathrm{A}$ to $\mathrm{C}$. The transmission failure rate of this experiment is $1.11 \%$. The average success transmission time (in seconds) increases slightly as the received signal strength decreases in all connections. The average transmission time of 178 success transmissions is about $2.949 \mathrm{sec}-$ onds. 
Table 1 A List of the Short Message Commands

\begin{tabular}{|c|l|}
\hline \multicolumn{1}{|c|}{ Short Message Commands } \\
\hline+ CSMS & Select Message Service \\
\hline+ CNMA & New Message Acknowledgement \\
\hline+ CPMS & Preferred Message Storage \\
\hline+ CMGF & Preferred Message Format \\
\hline+ CSAS & Save Settings \\
\hline+ CDES & Restore Settings \\
\hline+ CSDH & Show Text Mode Parameters \\
\hline+ CNMI & New Message Indication \\
\hline+ CMGR & Read Message \\
\hline+ CMGL & List Message \\
\hline+ CMGS & Send Message \\
\hline+ CMGW & Write Message to Memory in the SIM card \\
\hline+ CMSS & Send Message from Storage \\
\hline+ CSMP & Set Text Mode Parameters \\
\hline+ CMGD & Delete Message \\
\hline+ CSCA & Service Center Address \\
\hline+ CSCB & Select Cell Broadcast Message Types \\
\hline+ WCBM & Cell Broadcast Message Identifiers \\
\hline+ WMSC & Message Status Modification \\
\hline+ WMGO & Message Overwriting \\
\hline
\end{tabular}

Table 2 Average Transmission Time of Short Messages

\begin{tabular}{|c|c|c|c|}
\hline \multirow{2}{*}{$\begin{array}{c}\text { Received sig- } \\
\text { nal strength } \\
(\mathrm{dBm})\end{array}$} & $\begin{array}{c}\text { Average transmission time (seconds) } \\
\text { Connection } \\
\text { A to itself }\end{array}$ & $\begin{array}{c}\text { Route con- } \\
\text { nection } \\
\text { A to B }\end{array}$ & $\begin{array}{c}\text { Route } \\
\text { connection } \\
\text { A to C }\end{array}$ \\
\hline-100 & 3 & 5 & $5^{*}$ \\
\hline-90 & 2.5 & 4 & 5 \\
\hline-80 & 2.1 & 3.1 & 4.6 \\
\hline-70 & 2 & 2.2 & 2.3 \\
\hline-60 & 2 & 2.2 & 2.3 \\
\hline-50 & 2 & 2.1 & 2.1 \\
\hline
\end{tabular}

*Two short message are lost and the data is got by averaging the 8 success transmissions.

\section{DISCUSSION}

A portable TeleAlarm device design for independent living is discussed in detail in this work. The design is focused on the fast transmission of an emergency message in a low-cost way. The user could send emergency messages for help to his/her friends and relatives even to an emergency department, only if a PC available to download the received short messages from an MS.

The major disadvantage of the design is that the users have to stay at home or a place indicated in the short messages to make themselves be easily found in an emergency since the design does not provide a location information. It restricts the practical use of the design. There are two ways to overcome this problem theoretically. One is to compare the signal strength of the TeleAlarm device to a set of vicinal BSs [14], and the other is to use global positioning system (GPS) [15]-[17]. The signal strength method highly depends on the services of a network operator. On the other hand, if a GSM module has the build-in GPS to provide the location information, then it is possible to add the location information to the short messages. Such a GSM module is available. However, the size of the GSM module is too large to be portable.

\section{ACKNOWLEGEMENT}

This work is supported by the National Science Council under Contract NSC 90-2213E-262-008.

\section{REFERENCES}

1. Kaye LW: Telemedicine: Extension to home care, Telemedicine J. 1997; 3: 243-246.

2. Bronzino JD and Gover J: Medical technology: A solution to the health care cost problem, IEEE Eng. Med. Biol. Mag. 1994; 7; 313-315.

3. Ruggiero C, Giacomini M, and Sacile R: A multimedial environment for elderly health care: A local experience at the Savona, IEEE 0-7803-2050-6/94 1994.

4. Rodriguez MJ, Arredondo MT, del Pozo F, Gomez EJ, Martinez A, and Dopico A: A home tele-care management system, Computers in Cardiology 1994; 433-436.

5. Lee RG, Chen $\mathrm{HS}$, Lin $\mathrm{CC}$, Chang $\mathrm{KC}$, and Chen $\mathrm{JH}$ : Home telecare system using cable television plants - An experimental field trial, IEEE Trans. Inform. Technol. Biomed. 2000; 4: 37-44.

6. Brownsell SJ, Williams G, Bragg R, Catlin P, and Carlier J: Future system for remote health care, Journal of Telemedicine and Telecare 1999; 5 : 141152.

7. Tang $P$ and Venables T: Smart homes and telecare for independent living, Journal of Telemedicine and Telecare 2000; 6: 8-14.

8. Williams G, Doughty K, and Bradley DA: Safety and risk issues in using telecare, Journal of Telemedicine and Telecare 2000; 6: 249-262.

9. Robert, Habib Istepanian S: Modeling of GSMbased mobile telemedical system, Proceedings of the 20th Annual International Conference of the IEEE Engineering in Medicine and Biology Society 1998; 20: $1166-1169$. 
10. Woodward B, Istepanian RSH, and Richsrds CI: Design of a telemedicine system using a mobile telephone, IEEE Trans. Inform. Technol. Biomed. 2001; 5: 13-15.

11. Thanos A, Economakos G, Papakonstantinou G, Tsanakas P, and Nikolaidis L: An open system for ECG telemedicine and telecare, Computers in Cardiology 1998; 209-212.

12. Peersman G, Cvetkovic S, Griffiths $P$, and Spear H: The global system for mobile communications short message service," IEEE Personal Communications $2000 ; 15-23$.

13. Sollesei S, di Tria P, and Morena G: Short message service based applications in the GSM network, IEEE Transactions on Education 1999; 42: 939-943. 14. Drane C, Macnaughtan M, and Scott C: Positioning GSM telephone, IEEE Communications Mag. 1998; 46-59.

15. Allen JJ, et al.: Integrated micro-electro-mechanical sensor development for inertial applications, IEEE Position location and Navigation Symposium 1998; 9-16.

16. Brown J: Ring laser gyro/global positioning system navigation improvement program plus, IEEE Position location and Navigation Symposium 1996; 541-548. 